U.S. Department of the Interior

U.S. Geological Survey

\title{
History of Movement of the Clear Creek Forks Landslide, Clear Creek County, Colorado
}

\author{
W.Z. Savage, ${ }^{1}$ J.A. Coe, ${ }^{1}$ D.J. Varnes, ${ }^{1}$ R.K. Streufert, ${ }^{2}$ and J.W. Godt ${ }^{1}$ \\ 1 U.S. Geological Survey \\ MS 966, Box 25046 \\ Denver Federal Center \\ Denver, CO 80225 USA \\ 2 Colorado Geological Survey \\ 1313 Sherman Street \\ Denver, CO 80203 USA
}

\section{Open-File Report 98-150}

This report is preliminary and has not been reviewed for conformity with U.S. Geological Survey editorial standards or with the North American Stratigraphic Code. Any use of trade, product, or firm names is for descriptive purposes only and does not imply endorsement by the U.S. Government. 
Abstract: The Clear Creek Forks landslide near Central City, Colorado, threatens a major highway junction in Colorado's Front Range. Movement of the landslide was first reported during the mid-1940's when highway engineers noted deformation in bridges at the base of the landslide. Displacements of fixed points on the landslide have been surveyed at various times over the past 46 years. Displacement rates ranged up to $0.6 \mathrm{~m} / \mathrm{yr}$ in the early 1950 's but currently appear to be decreasing or negligible. However, ongoing reconfiguration of the highway junction, and planned removal of a bridge at the base of the landslide in the spring of 1998, raise the possibility of renewed movement.

\section{Introduction}

The Clear Creek Forks landslide is an unstable rock and colluvial slope at the junction of U.S. Highway 6 and Colorado State Highway 119 in Clear Creek County, Colorado (Fig. 1). The bedrock is gneiss of Precambrian age (Sheridan and Marsh, 1976). The foliation is locally irregular, but in the vicinity of the slide it generally strikes west and dips north at about the same angle as the surface of the ground (Fig. 2). This is probably the primary geologic factor influencing stability of the slope.

From its geomorphic expression, the landslide appears to be a reactivated older slide. It has an estimated volume of $300,000 \mathrm{~m}^{3}$ and for many years has slowly pushed U.S. Highway 6 and two bridges at its base toward Clear Creek, a major stream draining the Front Range of Colorado. This landslide threatens an important junction in a major transportation corridor, a junction that is currently being reconfigured by the Colorado Department of Transportation (CDOT, formerly the Colorado State Highway Department).
Movement of the landslide was first reported during the mid-1940's when highway engineers noted deformation in the highway (Fig. 3) and in bridges F15T and F15V (built in the late 1930's) spanning Clear Creek at the base of the landslide (D.J. Varnes, U.S. Geological Survey, unpub. report 1952). The bridge deformation, which was clearly related to movement of the landslide, was expressed by cracking of the abutments, northward tilting of the rockers (Fig. 4) supporting the bridge stringers, and complete closure of the original $7-9 \mathrm{~cm}$ clearance between ends of the stringers and the abutments of both bridges. During redecking of bridge F15T in 1985 the tipped rockers supporting the stringers were completely encased in new concrete, and cracks in abutments and wing walls were repaired (Lou Kingsley, CDOT, pers. commun. 3/24/98). As part of the ongoing highway reconfiguration, it is planned that the north abutment, stringers, and decking of bridge F15T will be removed in the spring of 1998. 
In this report, we describe the movement history of the landslide, as documented by the surveying of fixed points on the landslide surface at various times from the early 1950 's to the present. Reconstructing the history of movement will help in assessing any renewed movement that may be caused by road construction and bridge removal.

\section{Surveying History and Procedures}

The first survey to monitor the slide was made by the Colorado State Highway Department, which set a survey pin in the highway roadbed and four striped wands up the center of the slide in May 1951. Thirty to sixty centimeters of movement were measured during the following year. In 1952, the U.S. Geological Survey (USCS) began measurements of points on the slide, using triangulation from known survey stations along the Clear Creek County line on the ridge over the tunnel north of bridge F15T (points A and B, Fig. 5). Others points (known as "D" points, Figs. 2 and 5) were added and measurements were made six times until June 1953. Additional measurements were made in April and May 1955. In 1975, after electronic distance measuring equipment (EDM) was acquired, more observing stations were set on the ridge and measurements by triangulation and trilateration were made on old points, where they could be recovered, and on some new points. Measurements were again discontinued until 1987, when the Colorado Geological Survey established five new points (known as "R" points, Figs. 2 and 5) and remeasured their positions from 1990 to 1996 using a 'total station' which is a combination theodolite and EDM. Starting in early 1996, the USGS added four new "R" points, and resumed measurements of all other recoverable points using a total station. From mid1996 to present, the USGS has surveyed most points using differential Global Positioning System (GPS) techniques.

Our best estimate of the average error associated with all triangulation, EDM, and total station survey observations is about $0.1 \mathrm{~m}$ in easting and northing and $0.15 \mathrm{~m}$ in elevation. The average error of GPS observations is estimated to be about 0.02 $\mathrm{m}$ in easting and northing and about 0.03 $m$ in elevation.

\section{Movement History}

Measured displacements provide, for some points, a 46-year record of movement and reveal that the landslide has moved down and generally toward the north. The measured displacement data are given in Tables 1,2 , and 3 , and shown graphically in Figures 6, 7, and 8. Points with labels beginning with $D$ have the longest record of survey observations, from 1951 to 1997. Measured displacements of $D$ points with complete sets of survey observations (points D2, D5, and D6 in Fig. 6a, and D2 and D5 in Fig. 6b), suggest that most of the horizontal and vertical movement occurred prior to 1976. Displacement of $D$ points to the north over the 25-year period prior to 1976, ranged from near zero for points D6 and D10 on or outside the boundary of the landslide, to about $1.5 \mathrm{~m}$ for points D2 and D3 along the central portion of the slide. About half of the total cumulative, pre1976 , north displacements occurred in the first years of monitoring. (1951-52). Present rates of north displacement of $D$ points appear to have decreased or become negligible (Fig. 6a). Vertical displacements are $50-70 \%$ of corresponding north displacements and show a similar decreasing trend (Fig. 6b). The post-1976 record of movement is further documented by displacements of R points (Fig. 7), which were set in 1987. At first glance, it appears that $R$ points have been displaced to the 
north by as much as $0.4 \mathrm{~m}$, and to the east and vertically downward by as much as 0.2 $\mathrm{m}$. However, surveying observations before 1996 were made using a total station and hand-held range rod, whereas observations after 1996 were predominantly made using more accurate GPS techniques. Taking observing errors into account, we conclude that since 1987 the east and vertical displacements are near zero, and that north displacements are in the $0.15-$ to $0.25-\mathrm{m}$ range.

The upper portion of the slide has moved slightly toward the northeast, whereas the lower portion moved almost directly north (Fig 8). Vectors of cumulative north displacements in the central and lower portion of the slide are focused almost directly toward bridge F15T (Fig. 8).

\section{Conclusions}

This report documents roughly 46 years of movement at the Clear Creek Forks landslide from a series of measurements made in the 1950's, 1970's and 1990's.

Movement was first recognized in the mid1940 's, shortly after excavation for the current alignment of U.S. Highway 6 at the base of the landslide. Measurements of displacements of fixed survey points were begun in the early 1950 's. Within the first 2 years of monitoring (1951-52), displacements of points along the central portion of the landslide reached a maximum of about $0.6 \mathrm{~m} / \mathrm{yr}$. Since 1952, movement rates of the landslide (as indicated by the $D$ points in Fig. 6) have decreased. Movement rates since 1987 (as indicated by $R$ points in Fig. 7) have generally been less than 0.03 $\mathrm{m} / \mathrm{yr}$.

The junction of U.S. Highway 6 and Colorado State Highway 119 is currently being reconfigured and most of bridge F15T, at the base of the slide, is scheduled for removal in the spring of 1998. Results from past and continued USGS monitoring will be used to identify, and place into historical context, any movements that may result from bridge removal and road construction.

\section{References}

Sheridan, D.M. \& Marsh, S.P. 1976. Geologic map of the Squaw Pass Quadrangle, Clear Creek, Jefferson, and Gilpin counties. U.S. Geological Survey Geologic Quadrangle Map 1337, 1:24,000 scale. 
Table 1. East and North displacements of D points (in meters).

[East and North values are given relative to Point B.]

\begin{tabular}{|c|c|c|c|c|c|c|}
\hline DATE & \multicolumn{2}{|c|}{ DAYS STATION } & EAST & NORTH & DISP. E & DISP. N \\
\hline $05 / 31 / 51$ & 0 & D1 & -45.74 & -91.13 & 0.00 & 0.00 \\
\hline $06 / 07 / 51$ & 7 & D1 & -45.71 & -91.08 & 0.03 & 0.05 \\
\hline $06 / 14 / 51$ & 14 & D1 & -45.68 & -91.08 & 0.06 & 0.05 \\
\hline $06 / 21 / 51$ & 21 & D1 & -45.77 & -91.07 & -0.03 & 0.06 \\
\hline $06 / 28 / 51$ & 28 & D1 & -45.71 & -90.99 & 0.03 & 0.14 \\
\hline $07 / 20 / 51$ & 50 & D1 & -45.74 & -90.95 & -0.00 & 0.18 \\
\hline $08 / 06 / 51$ & 67 & D1 & -45.72 & -90.90 & 0.02 & 0.23 \\
\hline $10 / 22 / 51$ & 144 & D1 & -45.79 & -90.79 & -0.05 & 0.34 \\
\hline $12 / 06 / 51$ & 189 & D1 & -45.81 & -90.86 & -0.07 & 0.27 \\
\hline $03 / 18 / 52$ & 292 & D1 & -45.78 & -90.76 & -0.04 & 0.37 \\
\hline $04 / 03 / 52$ & 308 & D1 & -45.80 & -90.81 & -0.06 & 0.32 \\
\hline $05 / 31 / 51$ & 0 & D2 & -9.49 & -124.79 & 0.00 & 0.00 \\
\hline $07 / 20 / 51$ & 50 & D2 & -9.55 & -124.62 & -0.06 & 0.17 \\
\hline $03 / 18 / 52$ & 292 & D2 & -9.56 & -124.31 & -0.07 & 0.48 \\
\hline $10 / 02 / 52$ & 490 & D2 & -9.58 & -124.01 & -0.09 & 0.78 \\
\hline $06 / 15 / 53$ & 746 & D2 & -9.59 & -123.98 & -0.10 & 0.81 \\
\hline $04 / 29 / 55$ & 1429 & D2 & -9.60 & -124.05 & -0.11 & 0.74 \\
\hline $06 / 26 / 75$ & 8792 & D2 & -9.63 & -123.39 & -0.14 & 1.40 \\
\hline 04/17/96 & 16393 & D2 & -9.64 & -123.41 & -0.15 & 1.38 \\
\hline $05 / 31 / 51$ & 0 & D3 & -17.71 & -180.12 & 0.00 & 0.00 \\
\hline $06 / 07 / 51$ & 7 & D3 & -17.68 & -180.11 & 0.03 & 0.01 \\
\hline $06 / 14 / 51$ & 14 & D3 & -17.70 & -179.98 & 0.01 & 0.14 \\
\hline $06 / 21 / 51$ & 21 & D3 & -17.67 & -179.98 & 0.04 & 0.14 \\
\hline $07 / 20 / 51$ & 50 & D3 & -17.69 & -179.93 & 0.02 & 0.19 \\
\hline $08 / 06 / 51$ & 67 & D3 & -17.68 & -179.87 & 0.03 & 0.25 \\
\hline $10 / 22 / 51$ & 144 & D3 & -17.71 & -179.65 & -0.00 & 0.47 \\
\hline $12 / 06 / 51$ & 189 & D3 & -17.74 & -179.64 & -0.03 & 0.48 \\
\hline $03 / 18 / 52$ & 292 & D3 & -17.74 & -179.67 & -0.03 & 0.45 \\
\hline 04/03/52 & 308 & D3 & -17.71 & -179.65 & -0.00 & 0.47 \\
\hline $01 / 12 / 53$ & 592 & D3 & -17.73 & -179.31 & -0.02 & 0.81 \\
\hline $06 / 15 / 53$ & 746 & D3 & -17.72 & -179.26 & -0.01 & 0.86 \\
\hline $06 / 26 / 75$ & 8792 & D3 & -17.67 & -178.67 & 0.04 & 1.45 \\
\hline $05 / 31 / 51$ & 0 & D4 & -9.32 & -245.59 & 0.00 & 0.00 \\
\hline $06 / 14 / 51$ & 14 & D4 & -9.35 & -245.53 & -0.03 & 0.06 \\
\hline $06 / 21 / 51$ & 21 & D4 & -9.35 & -245.53 & -0.03 & 0.06 \\
\hline $06 / 28 / 51$ & 28 & D4 & -9.31 & -245.38 & 0.01 & 0.21 \\
\hline $07 / 20 / 51$ & 50 & D4 & -9.35 & -245.53 & -0.03 & 0.06 \\
\hline $08 / 06 / 51$ & 67 & D4 & -9.27 & -245.27 & 0.05 & 0.32 \\
\hline $10 / 22 / 51$ & 144 & D4 & -9.30 & -245.12 & 0.02 & 0.47 \\
\hline $12 / 06 / 51$ & 189 & D4 & -9.44 & -245.26 & -0.12 & 0.33 \\
\hline $03 / 18 / 52$ & 292 & D4 & -9.34 & -245.32 & -0.02 & 0.27 \\
\hline $04 / 03 / 52$ & 308 & D4 & -9.30 & -245.17 & 0.02 & 0.42 \\
\hline $05 / 13 / 52$ & 348 & D4 & -9.32 & -245.11 & -0.00 & 0.48 \\
\hline $05 / 28 / 52$ & 363 & D4 & -9.30 & -245.10 & 0.02 & 0.49 \\
\hline $08 / 27 / 52$ & 454 & D4 & -9.21 & -244.81 & 0.11 & 0.78 \\
\hline $10 / 02 / 52$ & 490 & D4 & -9.21 & -244.78 & 0.11 & 0.81 \\
\hline $01 / 12 / 53$ & 592 & D4 & -9.29 & -244.82 & 0.03 & 0.77 \\
\hline $06 / 15 / 53$ & 746 & D4 & -9.20 & -244.75 & 0.12 & 0.84 \\
\hline
\end{tabular}


Table 1. East and North displacements of D points (in meters)-Continued [East and North values are given relative to Point B.]

\begin{tabular}{|c|c|c|c|c|c|c|}
\hline DATE & DAYS & STATION & EAST & NORTH & DISP. E & DISP. N \\
\hline $05 / 03 / 55$ & 1433 & D4 & -9.25 & -244.91 & 0.07 & 0.68 \\
\hline $05 / 02 / 96$ & 16408 & D4 & -9.26 & -243.98 & 0.06 & 1.61 \\
\hline $05 / 13 / 52$ & 348 & D5 & 36.87 & -272.98 & 0.00 & 0.00 \\
\hline $05 / 28 / 52$ & 363 & D5 & 36.90 & -272.89 & 0.03 & 0.09 \\
\hline $08 / 27 / 52$ & 454 & D5 & 36.93 & -272.76 & 0.06 & 0.22 \\
\hline $10 / 02 / 52$ & 490 & D5 & 36.90 & -272.80 & 0.03 & 0.18 \\
\hline $01 / 12 / 53$ & 592 & D5 & 36.90 & -272.72 & 0.03 & 0.26 \\
\hline $06 / 15 / 53$ & 746 & D5 & 36.95 & -272.69 & 0.08 & 0.29 \\
\hline $06 / 26 / 75$ & 8792 & D5 & 37.00 & -272.28 & 0.13 & 0.70 \\
\hline $11 / 06 / 97$ & 16961 & D5 & 37.06 & -272.49 & 0.19 & 0.49 \\
\hline $05 / 13 / 53$ & 713 & D6 & 46.25 & -192.00 & 0.00 & 0.00 \\
\hline $05 / 28 / 53$ & 728 & D6 & 46.26 & -192.03 & 0.01 & -0.03 \\
\hline $08 / 27 / 52$ & 454 & D6 & 46.26 & -191.99 & 0.01 & 0.01 \\
\hline $10 / 02 / 52$ & 490 & D6 & 46.26 & -191.99 & 0.01 & 0.01 \\
\hline $06 / 15 / 53$ & 746 & D6 & 46.26 & -191.99 & 0.01 & 0.01 \\
\hline $06 / 26 / 75$ & 8792 & D6 & 46.26 & -191.92 & 0.01 & 0.08 \\
\hline $04 / 17 / 96$ & 16393 & D6 & 46.32 & -192.00 & 0.06 & 0.00 \\
\hline $06 / 04 / 96$ & 16441 & D6 & 46.40 & -191.97 & 0.14 & 0.03 \\
\hline $05 / 28 / 52$ & 363 & D7 & -122.05 & -223.17 & 0.00 & 0.00 \\
\hline $08 / 28 / 52$ & 455 & D7 & -122.04 & -223.14 & 0.01 & 0.03 \\
\hline $10 / 02 / 52$ & 490 & D7 & -122.06 & -223.14 & -0.01 & 0.03 \\
\hline $01 / 12 / 53$ & 592 & D7 & -122.05 & -223.12 & -0.00 & 0.05 \\
\hline $06 / 15 / 53$ & 746 & D7 & -122.05 & -223.18 & 0.00 & -0.01 \\
\hline $05 / 28 / 52$ & 363 & D8 & -76.81 & -204.95 & 0.00 & 0.00 \\
\hline $08 / 28 / 52$ & 455 & D8 & -76.84 & -204.79 & -0.03 & 0.16 \\
\hline $10 / 02 / 52$ & 490 & D8 & -76.85 & -204.76 & -0.04 & 0.19 \\
\hline $01 / 12 / 53$ & 592 & D8 & -76.86 & -204.72 & -0.05 & 0.23 \\
\hline $06 / 15 / 53$ & 746 & D8 & -76.85 & -204.76 & -0.04 & 0.19 \\
\hline $11 / 07 / 97$ & 16962 & D8 & -76.95 & -204.34 & -0.14 & 0.61 \\
\hline $05 / 28 / 52$ & 363 & D9 & -50.17 & -241.43 & 0.00 & 0.00 \\
\hline $08 / 28 / 52$ & 455 & D9 & -50.21 & -241.22 & -0.04 & 0.21 \\
\hline $10 / 02 / 52$ & 490 & D9 & -50.23 & -241.19 & -0.06 & 0.24 \\
\hline $01 / 12 / 53$ & 592 & D9 & -50.24 & -241.19 & -0.07 & 0.24 \\
\hline $06 / 15 / 53$ & 746 & D9 & -50.22 & -241.17 & -0.05 & 0.26 \\
\hline $05 / 28 / 52$ & 363 & D10 & -17.90 & -322.17 & 0.00 & 0.00 \\
\hline $08 / 27 / 52$ & 454 & D10 & -17.89 & -322.01 & 0.01 & 0.16 \\
\hline $10 / 02 / 52$ & 490 & D10 & -17.92 & -322.17 & -0.02 & -0.00 \\
\hline $01 / 12 / 53$ & 592 & D10 & -17.95 & -322.05 & -0.05 & 0.12 \\
\hline $06 / 15 / 53$ & 746 & D10 & -17.87 & -322.10 & 0.03 & 0.07 \\
\hline 06/04/96 & 16441 & D10 & -17.79 & -322.38 & 0.11 & -0.21 \\
\hline $12 / 12 / 96$ & 16632 & D10 & -17.92 & -322.35 & -0.02 & -0.18 \\
\hline $10 / 02 / 52$ & 490 & D11 & -18.16 & -198.33 & 0.00 & 0.00 \\
\hline $06 / 15 / 53$ & 746 & D11 & -17.96 & -197.83 & 0.20 & 0.50 \\
\hline $09 / 25 / 97$ & 16919 & D1 & -18.07 & -197.79 & 0.09 & 0.54 \\
\hline $06 / 20 / 75$ & 8786 & D1 & -9.61 & -155.30 & 0.00 & 0.00 \\
\hline $04 / 17 / 96$ & 16393 & D12 & -9.62 & -155.28 & -0.01 & 0.02 \\
\hline
\end{tabular}


Table 2. Vertical displacements of D points (in meters)

[Elevation values given are relative to sea level.]

\begin{tabular}{rrrrr}
\hline DATE & DAYS & STATION & ELEV & DIFF. ELEV \\
\hline $05 / 28 / 52$ & 15 & D2 & 2107.81 & 0.00 \\
$08 / 27 / 52$ & 106 & D2 & 2107.70 & -0.11 \\
$10 / 02 / 52$ & 142 & D2 & 2107.67 & -0.14 \\
$01 / 12 / 53$ & 244 & D2 & 2107.60 & -0.21 \\
$06 / 15 / 53$ & 398 & D2 & 2107.65 & -0.16 \\
$06 / 26 / 75$ & 8444 & D2 & 2107.22 & -0.59 \\
$04 / 24 / 96$ & 16052 & D2 & 2107.06 & -0.75 \\
$05 / 13 / 52$ & 0 & D3 & 2146.44 & 0.00 \\
$05 / 28 / 52$ & 15 & D3 & 2146.41 & -0.03 \\
$08 / 28 / 52$ & 107 & D3 & 2146.27 & -0.17 \\
$10 / 02 / 52$ & 142 & D3 & 2146.28 & -0.16 \\
$01 / 12 / 53$ & 244 & D3 & 2146.19 & -0.25 \\
$06 / 15 / 53$ & 398 & D3 & 2146.26 & -0.18 \\
$06 / 26 / 75$ & 8444 & D3 & 2145.83 & -0.61 \\
$05 / 13 / 52$ & 0 & D4 & 2189.35 & 0.00 \\
$05 / 28 / 52$ & 15 & D4 & 2189.41 & 0.06 \\
$08 / 27 / 52$ & 106 & D4 & 2189.81 & 0.46 \\
$10 / 02 / 52$ & 142 & D4 & 2189.75 & 0.40 \\
$01 / 12 / 53$ & 244 & D4 & 2189.67 & 0.32 \\
$06 / 15 / 53$ & 398 & D4 & 2189.74 & 0.39 \\
$04 / 24 / 96$ & 16052 & D4 & 2188.37 & -0.98 \\
$09 / 05 / 97$ & 16551 & D4 & 2188.43 & -0.92 \\
$05 / 13 / 52$ & 0 & D5 & 2184.74 & 0.00 \\
$05 / 28 / 52$ & 15 & D5 & 2184.69 & -0.05 \\
$08 / 27 / 52$ & 106 & D5 & 2184.61 & -0.13 \\
$10 / 02 / 52$ & 142 & D5 & 2184.62 & -0.12 \\
$01 / 12 / 53$ & 244 & D5 & 2184.56 & -0.18 \\
$06 / 15 / 53$ & 398 & D5 & 2184.60 & -0.14 \\
$06 / 26 / 75$ & 8444 & D5 & 2184.35 & -0.39 \\
$12 / 11 / 97$ & 16648 & D5 & 2184.45 & -0.29 \\
$05 / 13 / 52$ & 0 & D6 & 2127.29 & 0.00 \\
$05 / 28 / 52$ & 15 & D6 & 2127.29 & 0.00 \\
$08 / 27 / 52$ & 106 & D6 & 2127.31 & 0.02 \\
$10 / 02 / 52$ & 142 & D6 & 2127.34 & 0.05 \\
\hline
\end{tabular}


Table 2. Vertical displacements of D points (in meters)-Continued [Elevation values given are relative to sea level.]

\begin{tabular}{rrrrrr}
\hline DATE & DAYS & STATION & ELEV & DIFF. ELEV \\
\hline $01 / 12 / 53$ & 244 & D6 & 2127.30 & 0.01 \\
$06 / 15 / 53$ & 398 & D6 & 2127.31 & 0.02 \\
$04 / 24 / 96$ & 16052 & D6 & 2127.31 & 0.02 \\
$09 / 05 / 96$ & 16186 & D6 & 2127.25 & -0.04 \\
$05 / 28 / 52$ & 15 & D7 & 2151.25 & 0.00 \\
$08 / 28 / 52$ & 107 & D7 & 2151.26 & 0.00 \\
$10 / 02 / 52$ & 142 & D7 & 2151.25 & -0.01 \\
$01 / 12 / 53$ & 244 & D7 & 2151.20 & -0.06 \\
$06 / 15 / 53$ & 398 & D7 & 2151.24 & -0.02 \\
$05 / 28 / 52$ & 15 & D8 & 2152.43 & 0.00 \\
$08 / 28 / 52$ & 107 & D8 & 2152.35 & -0.08 \\
$10 / 02 / 52$ & 142 & D8 & 2152.30 & -0.13 \\
$01 / 12 / 53$ & 244 & D8 & 2152.30 & -0.13 \\
$06 / 15 / 53$ & 398 & D8 & 2152.29 & -0.14 \\
$12 / 11 / 97$ & 16648 & D8 & 2152.01 & -0.42 \\
$05 / 28 / 52$ & 15 & D9 & 2180.75 & 0.00 \\
$08 / 28 / 52$ & 107 & D9 & 2180.65 & -0.10 \\
$10 / 02 / 52$ & 142 & D9 & 2180.56 & -0.19 \\
$01 / 12 / 53$ & 244 & D9 & 2180.54 & -0.21 \\
$06 / 15 / 53$ & 398 & D9 & 2180.57 & -0.18 \\
$05 / 28 / 52$ & 15 & D10 & 2233.82 & 0.00 \\
$08 / 27 / 52$ & 106 & D10 & 2233.86 & 0.04 \\
$10 / 02 / 52$ & 142 & D10 & 2233.86 & 0.04 \\
$01 / 12 / 53$ & 244 & D10 & 2233.71 & -0.11 \\
$06 / 15 / 53$ & 398 & D10 & 2233.85 & 0.03 \\
$06 / 04 / 96$ & 16093 & D10 & 2233.84 & 0.02 \\
$10 / 02 / 52$ & 142 & D11 & 2157.70 & 0.00 \\
$01 / 12 / 53$ & 244 & D11 & 2157.61 & -0.09 \\
$06 / 15 / 53$ & 398 & D11 & 2157.67 & -0.03 \\
$09 / 25 / 97$ & 16571 & D11 & 2177.24 & -0.46 \\
$06 / 20 / 75$ & 8438 & D12 & 2128.90 & 0.00 \\
$04 / 24 / 96$ & 16052 & D12 & 2128.82 & -0.08 \\
$06 / 04 / 96$ & 16093 & D12 & 2128.83 & -0.07 \\
$09 / 05 / 96$ & 16186 & D12 & 2128.86 & -0.04 \\
\hline & & & & & \\
\hline
\end{tabular}


Table 3. Displacements (in meters) of $R$ points

[East, North, and Elevation values are given relative to Point B.]

\begin{tabular}{|c|c|c|c|c|c|c|c|}
\hline DATE & STATION & EAST & NORTH & DISP. E & DISP. N & ELEV & DIFF.ELEV \\
\hline $05 / 04 / 90$ & $\mathrm{R} 1$ & -10.84 & -325.18 & 0.00 & 0.00 & & \\
\hline $10 / 19 / 90$ & R1 & -10.83 & -325.22 & 0.01 & -0.04 & 2233.05 & 0.00 \\
\hline $05 / 31 / 91$ & R1 & -10.79 & -325.20 & 0.05 & -0.02 & 2233.08 & 0.03 \\
\hline $04 / 24 / 96$ & R1 & -10.93 & -325.06 & -0.09 & 0.12 & 2233.02 & -0.03 \\
\hline $05 / 02 / 96$ & R1 & -10.77 & -325.10 & 0.07 & 0.08 & 2233.16 & 0.11 \\
\hline $06 / 04 / 96$ & R1 & -10.71 & -325.13 & 0.13 & 0.05 & 2233.10 & 0.05 \\
\hline 09/05/96 & R1 & -10.85 & -325.08 & -0.01 & 0.10 & 2233.14 & 0.09 \\
\hline $12 / 12 / 96$ & R1 & -10.85 & -325.07 & -0.01 & 0.11 & 2233.12 & 0.07 \\
\hline $09 / 02 / 97$ & R1 & -10.83 & -325.01 & 0.01 & 0.17 & 2233.14 & 0.09 \\
\hline $11 / 12 / 87$ & $\mathrm{R} 2$ & 1.40 & -256.36 & 0.00 & 0.00 & & 0.00 \\
\hline $05 / 04 / 90$ & R2 & 1.34 & -256.32 & -0.06 & 0.04 & & 0.00 \\
\hline $10 / 19 / 90$ & $\mathrm{R} 2$ & 1.22 & -256.35 & -0.18 & 0.01 & 2191.85 & 0.00 \\
\hline $05 / 31 / 91$ & R2 & 1.24 & -256.35 & -0.16 & 0.01 & 2191.81 & -0.04 \\
\hline $04 / 24 / 96$ & $\mathrm{R} 2$ & 1.26 & -256.24 & -0.14 & 0.12 & 2191.81 & -0.04 \\
\hline $05 / 02 / 96$ & $\mathrm{R} 2$ & 1.29 & -256.26 & -0.11 & 0.10 & 2191.86 & 0.01 \\
\hline $06 / 04 / 96$ & R2 & 1.31 & -256.27 & -0.09 & 0.09 & 2191.84 & -0.01 \\
\hline $09 / 05 / 96$ & $\mathrm{R} 2$ & 1.20 & -256.24 & -0.20 & 0.12 & 2191.89 & 0.04 \\
\hline $12 / 12 / 96$ & $\mathrm{R} 2$ & 1.19 & -256.23 & -0.21 & 0.13 & 2191.86 & 0.01 \\
\hline $12 / 12 / 96$ & $\mathrm{R} 2$ & 1.22 & -256.18 & -0.18 & 0.18 & 2191.89 & 0.04 \\
\hline $05 / 02 / 97$ & $\mathrm{R} 2$ & 1.20 & -256.17 & -0.20 & 0.19 & 2191.89 & 0.04 \\
\hline $11 / 12 / 87$ & R3 & -8.77 & -168.54 & 0.00 & 0.00 & & 0.00 \\
\hline $05 / 04 / 90$ & R3 & -8.76 & -168.29 & 0.01 & 0.25 & & 0.00 \\
\hline $10 / 19 / 90$ & R3 & -8.74 & -168.32 & 0.03 & 0.22 & 2142.15 & 0.00 \\
\hline $05 / 31 / 91$ & R3 & -8.75 & -168.32 & 0.02 & 0.22 & 2142.12 & -0.03 \\
\hline $04 / 2$ & R3 & -8.75 & .20 & 0.02 & 0.34 & 2142.11 & -0.04 \\
\hline $05 / 02 / 96$ & R3 & -8.71 & .23 & 0.06 & 0.31 & 2142.17 & 0.02 \\
\hline $06 / 04 / 96$ & R3 & -8.71 & -168.24 & 0.06 & 0.30 & 2142.12 & -0.03 \\
\hline $09 / 05 / 96$ & R3 & -8.78 & -168.19 & -0.01 & 0.35 & 2142.19 & 0.04 \\
\hline $12 / 12 / 96$ & R3 & -8.78 & -168.19 & -0.01 & 0.35 & 2142.16 & 0.01 \\
\hline $12 / 12 / 96$ & R3 & -8.77 & -168.18 & 0.00 & 0.36 & 2142.20 & 0.05 \\
\hline $05 / 02 / 97$ & R3 & -8.77 & -168.17 & 0.00 & 0.37 & 2142.18 & 0.03 \\
\hline $09 / 0$ & R3 & -8.76 & -168 & 0.01 & 0.37 & 2142.20 & 0.05 \\
\hline 187 & $\mathrm{R}$ & 33.87 & -17 & 0.00 & 0.00 & & 0.00 \\
\hline $05 / 04 / 90$ & R4 & 33.95 & -178.01 & 0.08 & 0.16 & & 0.00 \\
\hline $10 / 19 / 90$ & R4 & 33.78 & -178.10 & -0.09 & 0.07 & 2130.14 & 0.00 \\
\hline $05 / 31 / 91$ & R4 & 33.80 & -178.10 & -0.07 & 0.07 & 2130.10 & -0.04 \\
\hline $04 / 24 / 96$ & R4 & 33.93 & -177.92 & 0.06 & 0.25 & 2130.04 & -0.10 \\
\hline $05 / 02 / 96$ & R4 & 33.82 & -177.92 & -0.05 & 0.25 & 2130.15 & 0.01 \\
\hline $09 / 05 / 96$ & R4 & 33.66 & -178.00 & -0.21 & 0.17 & 2130.16 & 0.02 \\
\hline $12 / 12 / 96$ & R4 & 33.76 & -178.00 & -0.11 & 0.17 & 2130.15 & 0.01 \\
\hline $12 / 12 / 96$ & R4 & 33.76 & -177.96 & -0.11 & 0.21 & 2130.17 & 0.03 \\
\hline $05 / 02 / 97$ & R4 & 33.76 & -177.97 & -0.11 & 0.20 & 2130.15 & 0.01 \\
\hline $09 / 02 / 97$ & R4 & 33.76 & -177.97 & -0.11 & 0.20 & 2130.15 & 0.01 \\
\hline $11 / 12 / 87$ & R5 & -113.94 & -243.40 & 0.00 & 0.00 & & 0.00 \\
\hline $05 / 04 / 90$ & R5 & -114.06 & -243.16 & -0.12 & 0.24 & & 0.00 \\
\hline $10 / 19 / 90$ & R5 & 4.05 & -243.20 & -0.11 & 0.20 & 2171.02 & 0.00 \\
\hline $05 / 31 / 91$ & R5 & 4.02 & & -0.08 & 0.21 & 2171.00 & -0.02 \\
\hline $04 / 24 / 96$ & R5 & -114.09 & -243.08 & -0.15 & 0.32 & 2171.12 & 0.10 \\
\hline $05 / 02 / 96$ & R5 & -114.00 & -243.13 & -0.06 & 0.27 & 2171.04 & 0.02 \\
\hline $06 / 04 / 96$ & R5 & -114.00 & -243.14 & -0.06 & 0.26 & 2171.02 & 0.00 \\
\hline $09 / 05 / 96$ & R5 & -114.11 & -243.06 & -0.17 & 0.34 & 2171.06 & 0.04 \\
\hline $12 / 12 / 96$ & R5 & -114.11 & -243.05 & -0.17 & 0.35 & 2171.03 & 0.01 \\
\hline $12 / 12 / 96$ & R5 & -114.06 & -243.00 & -0.12 & 0.40 & 2171.06 & 0.04 \\
\hline $09 / 02 / 97$ & R5 & -114.06 & -243.00 & -0.12 & 0.40 & 2171.07 & 0.05 \\
\hline
\end{tabular}


Figure 1. Aerial view of the Clear Creek Forks landslide, the west part of the junction of U.S. Highway 6 and Colorado State Highway 119, and the confluence of Clear Creek and North Clear Creek. Small, white arrows in streams indicate direction of flow.

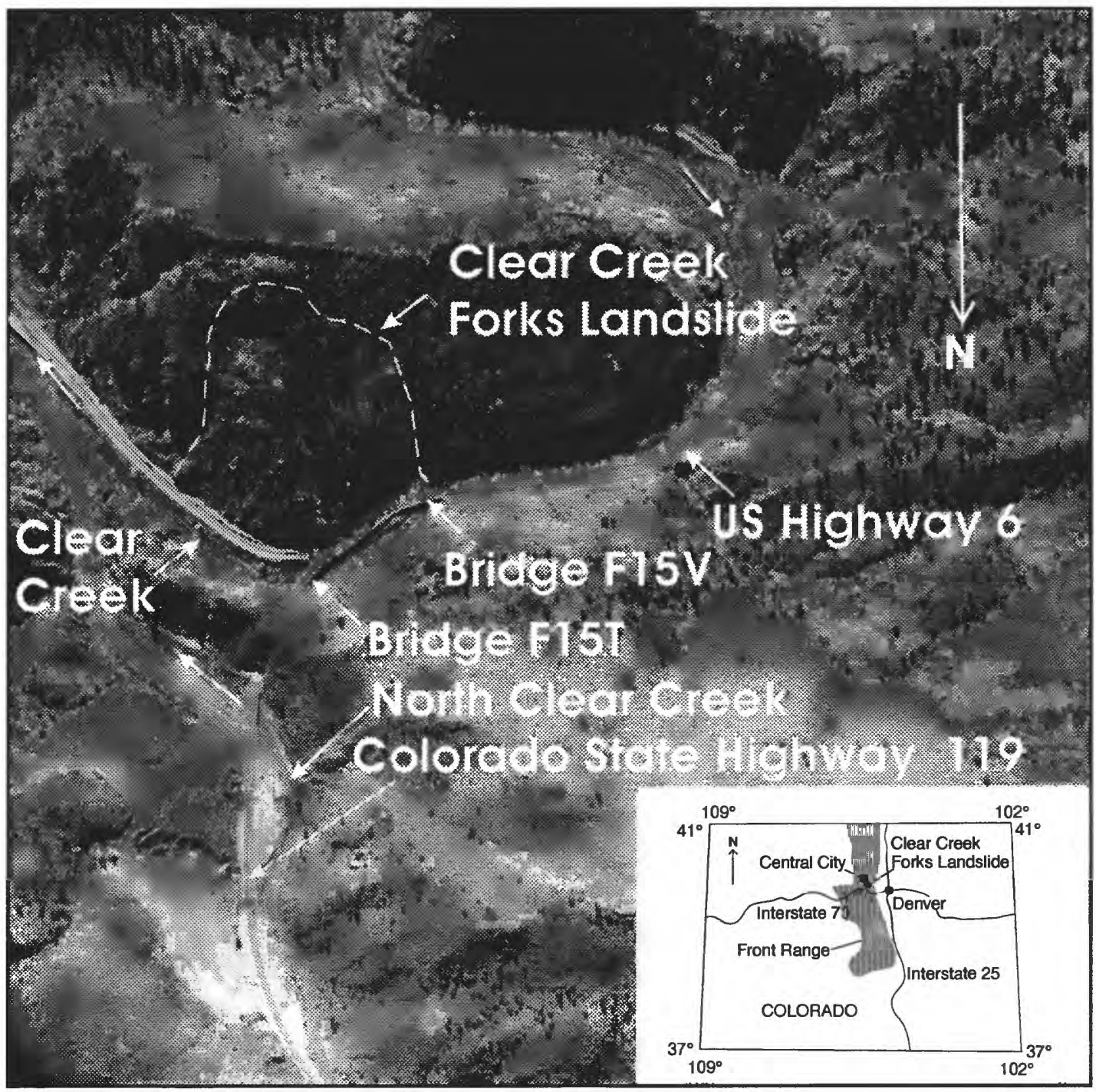

Figure 2. Photomosaic showing the landslide boundary and surveyed points. View is to the south. Scale varies in this perspective view. Vehicles on road give scale of foreground. Distances from the road to the top of the ridge are about $150 \mathrm{~m}$ vertical and $240 \mathrm{~m}$ horizontal. Photos taken May 31, 1996, by J.A. Coe. 


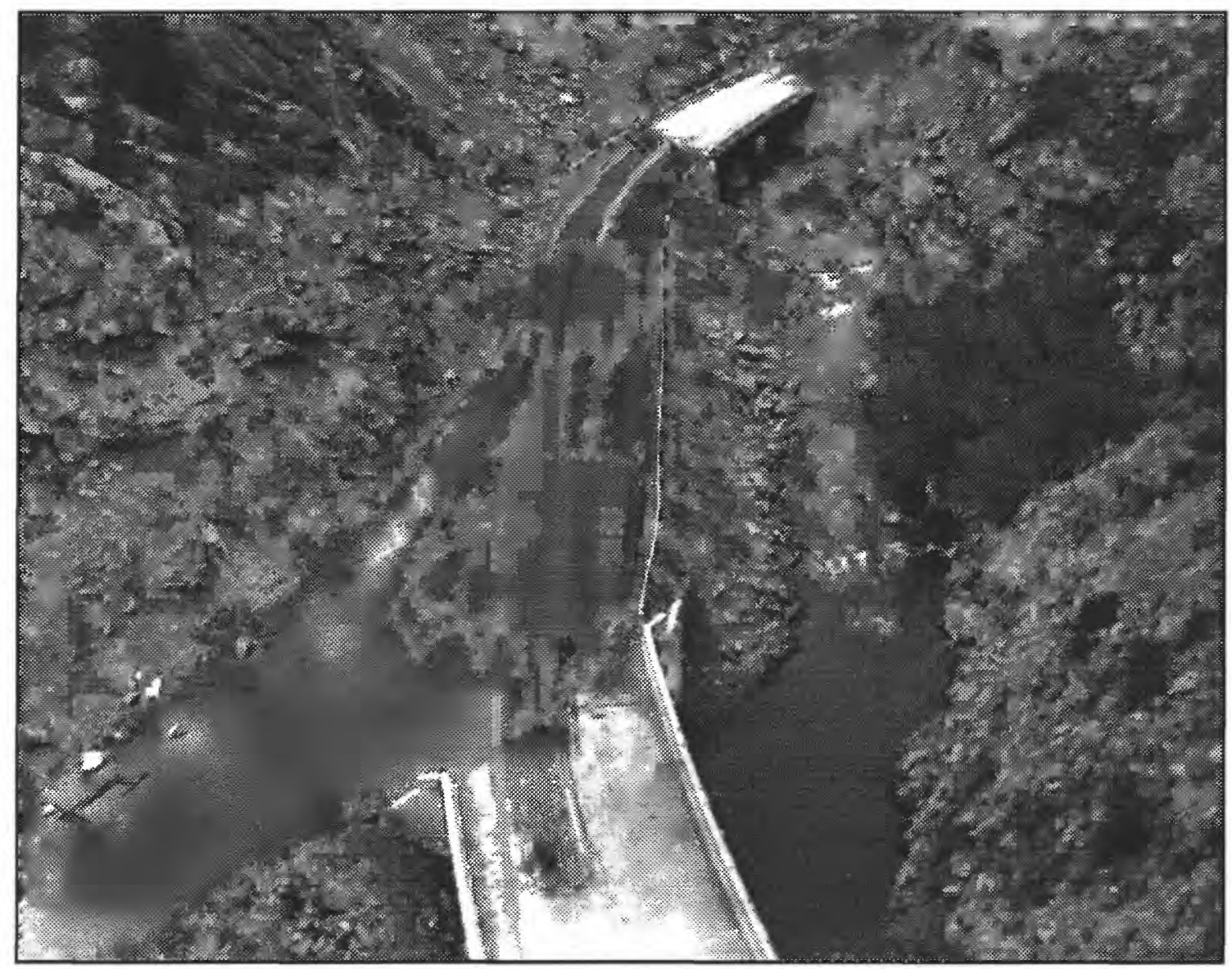

Figure 3. View of deformation in

State Highway 119 (now named U.S. Highway 6) and deformation of guardrail between bridge F15T, lower center, and bridge F15V, top.

Photo taken October 1951, by $D J$. Varnes.

Figure 4. Tipped rocker at the northwest corner of bridge F15V. Photo taken in early 1950's by DJ. Varnes.

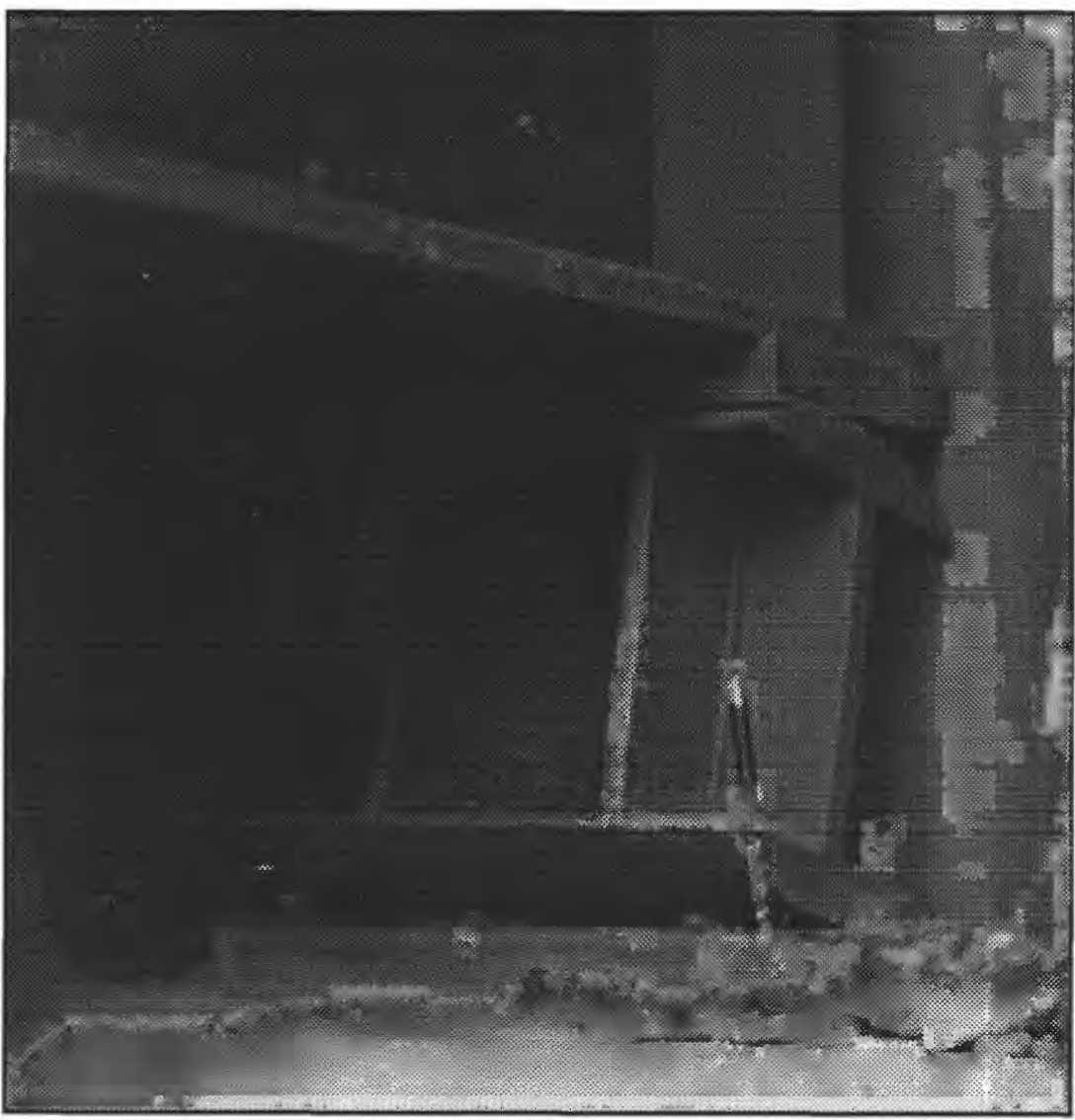




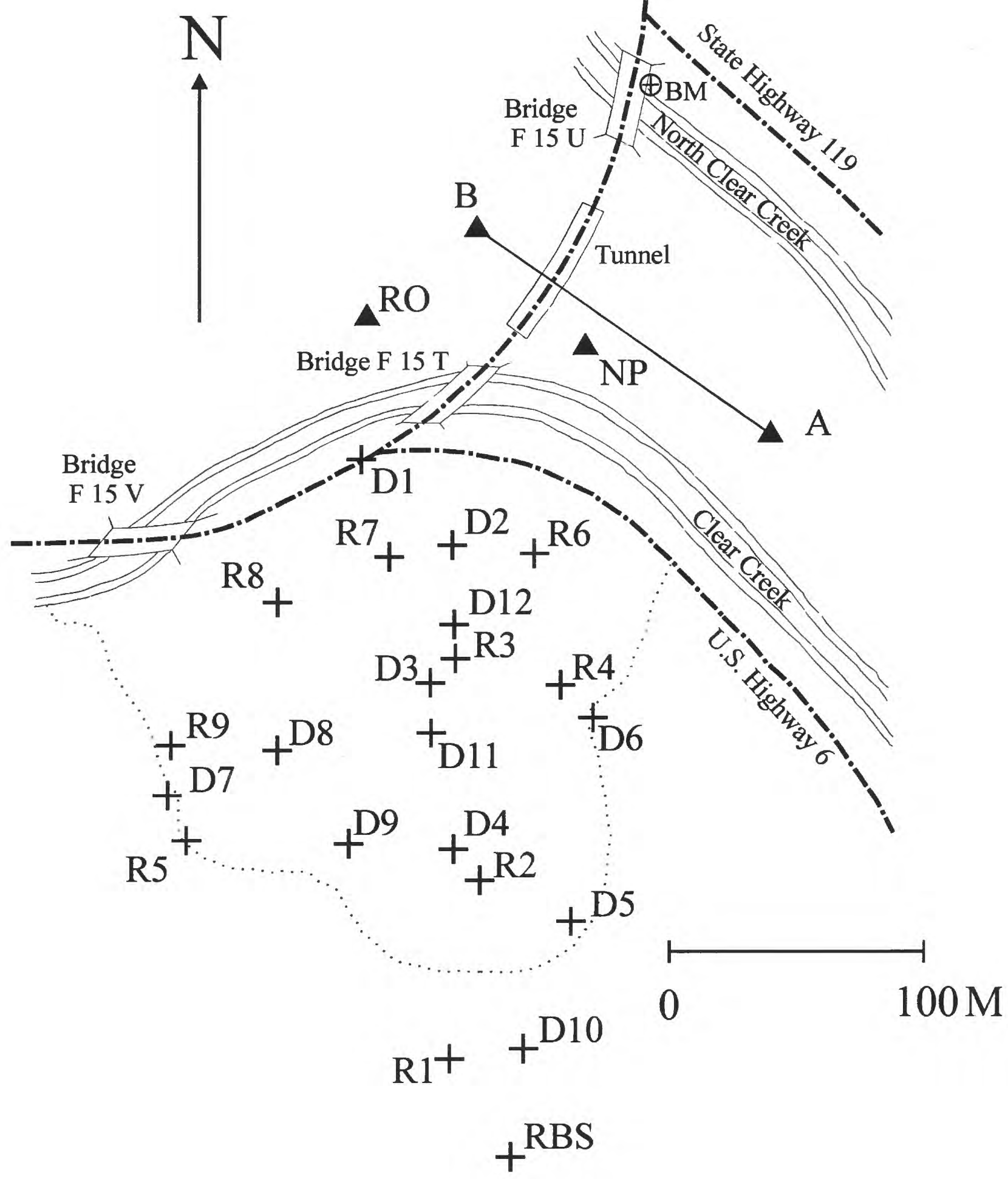

Figure 5. Map showing landslide boundary, triangulation baseline (line between points $A$ and $B$ ), and survey points on the landslide ( $D$ and $R$ points). Points NP, RO, and RBS have been used as base and backsight points for theodolite and EDM surveys in the 1980's and 1990's. 


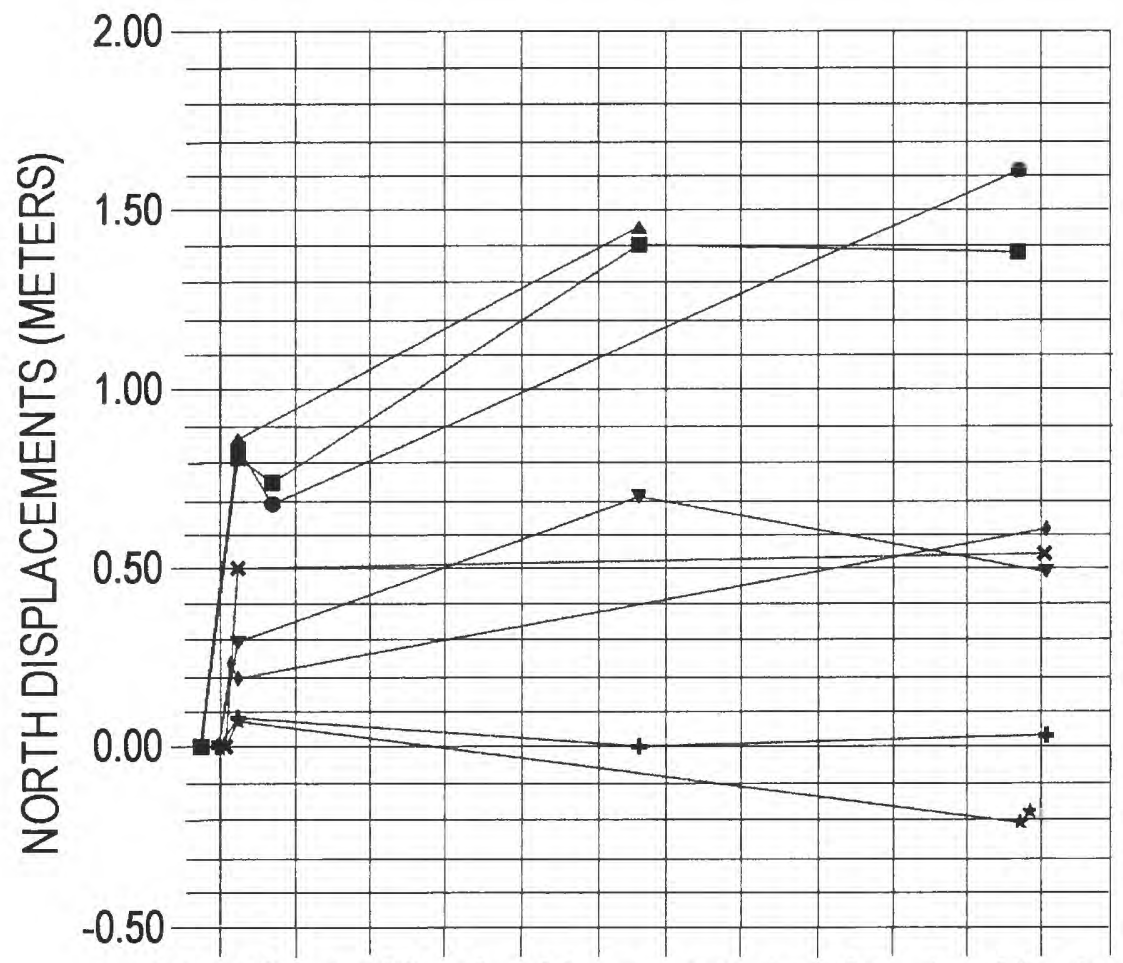

May-52 Jul-60 Oct-68 Jan-77 Mar-85 Jun-93 Aug-01

(a)

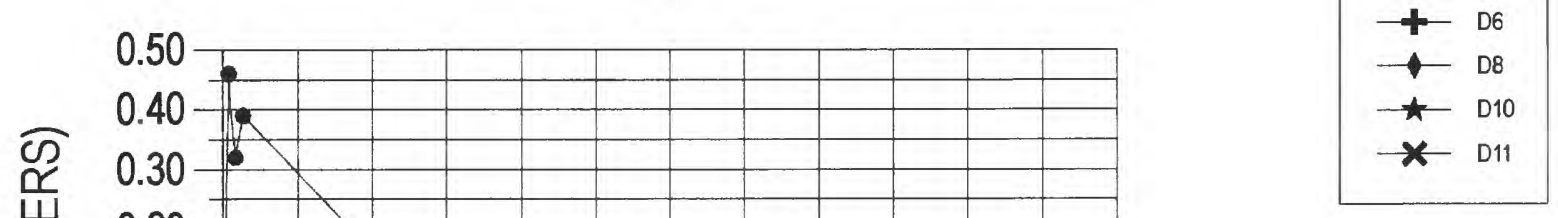

Figure 6. North (a) and vertical (b) components of displacements of " $D$ " survey points. Total cumulative displacements to the east over the entire monitoring period (46 years) are all less than $0.2 m$ and are not shown. 

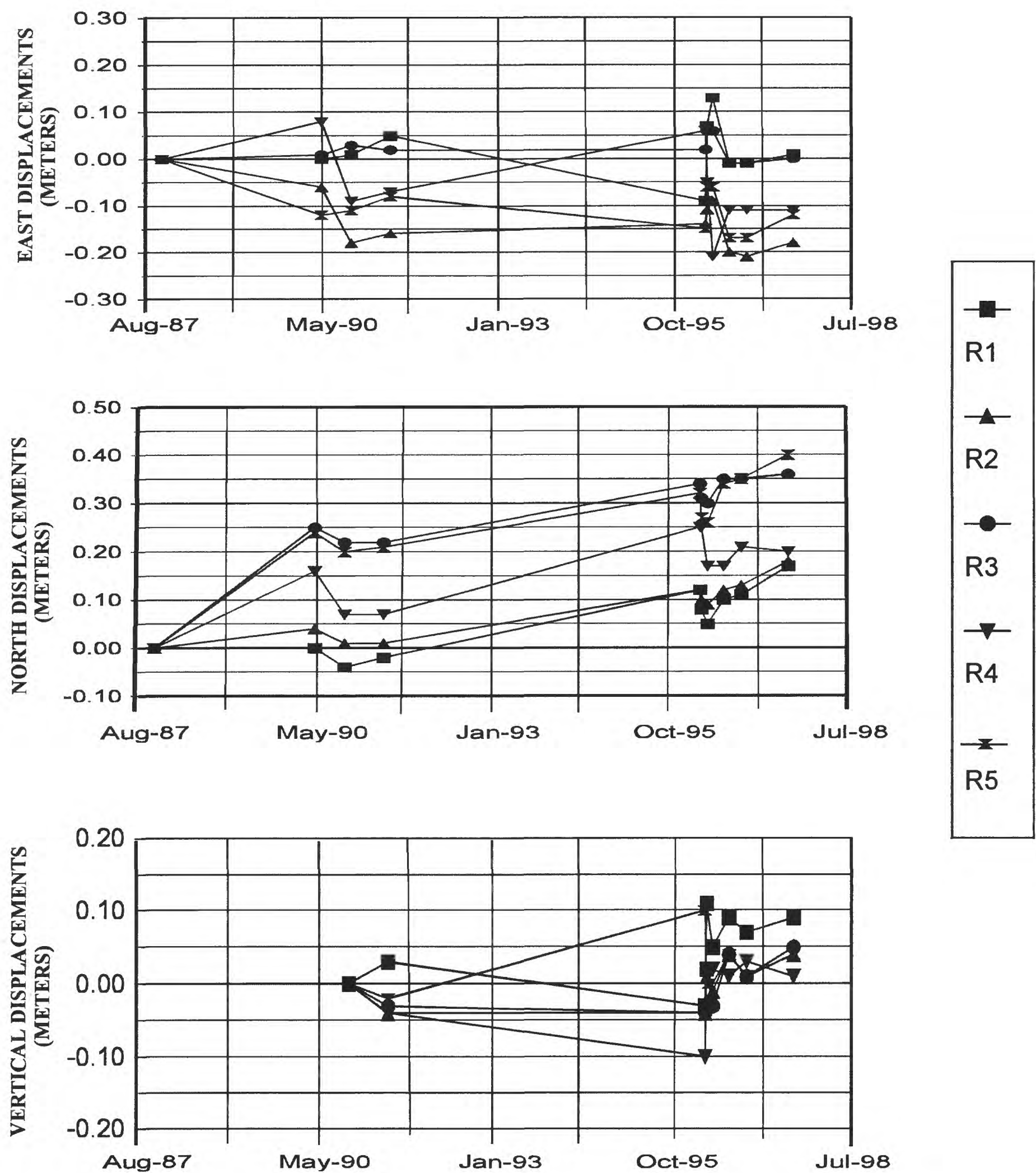

Figure 7. Displacements of " $R$ " survey points. Displacements of points R6-R9 are not shown because only one set of survey observations (1997) currently exists. 


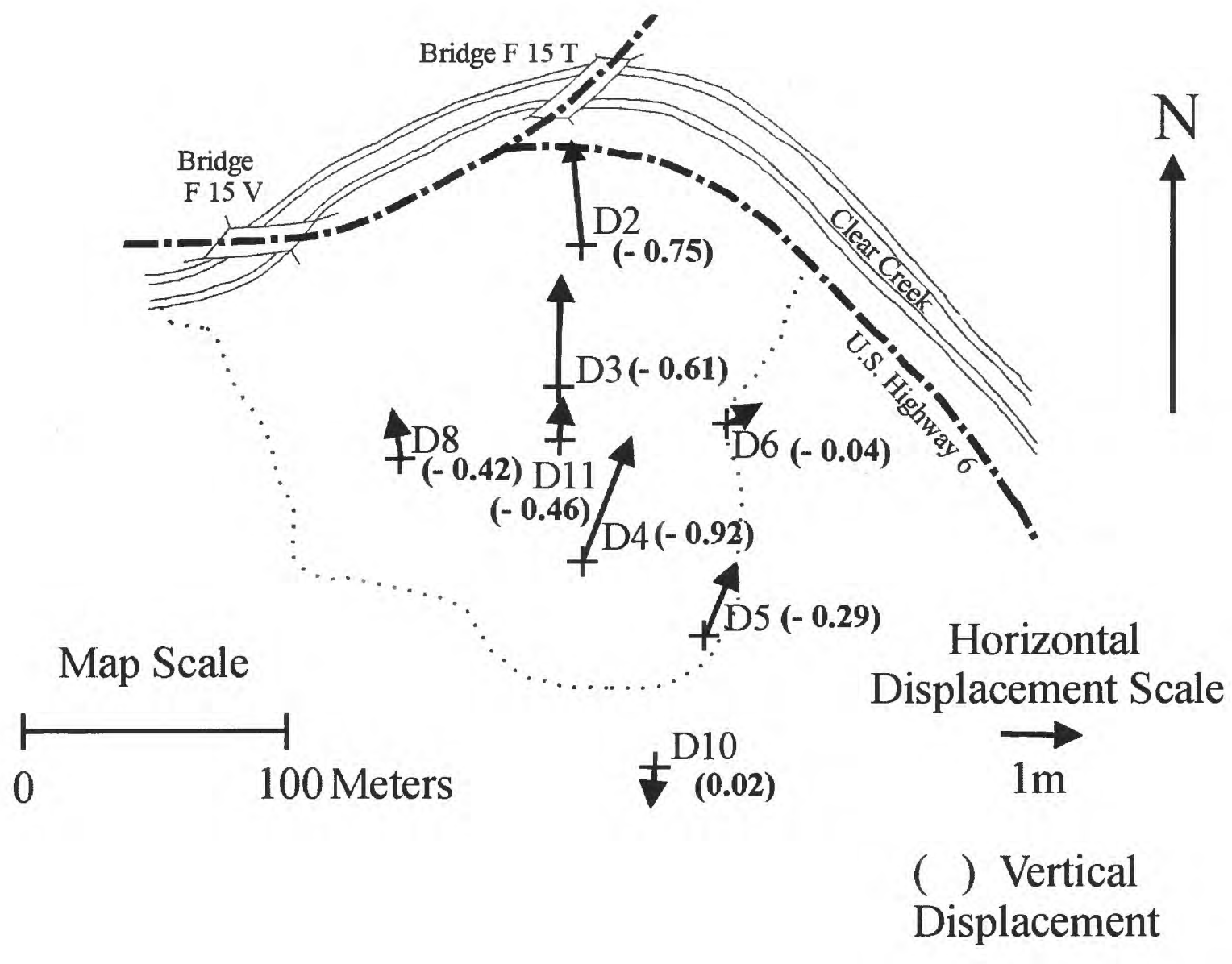

Figure 8. Map showing cumulative (1951-1997) displacement vectors for " $D$ " points shown in Figure 6. Vertical displacements are shown in parentheses. Units are meters and are designated with negative and positive numbers for downward and upward movement, respectively. 